\title{
A eficácia das intervenções farmacológicas e psicossociais para o tratamento do tabagismo: revisão da literatura
}

\author{
Cláudia Galvão Mazoni \\ Universidade Luterana do Brasil \\ Simone Fernandes \\ Serviço Nacional de Orientações e Informações sobre a Prevenção do Uso Indevido de Drogas - VIVAVOZ \\ Pollianna Sangalli Pierozan \\ Instituto de Desenvolvimento Educacional do Alto Uruguai \\ Taís Moreira \\ Luana Freese \\ Maristela Ferigolo \\ Helena M. T. Barros \\ Serviço Nacional de Orientações e Informações sobre a Prevenção do Uso Indevido de Drogas - VIVAVOZ
}

\begin{abstract}
Resumo
A cessação do tabagismo traz benefícios à saúde, pois indivíduos que param de fumar evitam a maioria das doenças associadas ao tabaco. Objetiva-se revisar as características fundamentais e eficácia das intervenções farmacológicas e psicossociais para o tratamento dos tabagistas, apresentando dados de revisões tipo meta-análises e de ensaios clínico randomizados. Intervenções farmacológicas como a terapia de reposição de nicotina e a bupropiona e intervenções psicossociais como a terapia cognitivo-comportamental e a intervenção motivacional face a face e por telefone demonstram eficácia. A farmacoterapia aumenta a chance de abstinência em 2 vezes e as intervenções psicossociais face a face ou por telefone em 1,5 a 2,5 vezes em relação a tabagistas recebendo intervenções controles. Estes dados fornecem subsídios para profissionais de saúde decidirem qual o melhor tratamento para o tabagista, informando as intervenções disponíveis, sua eficácia e o benefício de sua utilização.

Palavras-chave: tabaco; tabagismo; terapia; farmacoterapia
\end{abstract}

\begin{abstract}
Efficacy of pharmacological and no-pharmacological treatments for smoking cessation. Smoking cessation is associated to health benefits, because individuals who stop smoking will avoid most tobacco-related disorders. Our aim was to review the most important characteristics and the efficacy of the pharmacological and psychossocial treatments available for tobacco smokers, presenting meta-analysis and randomized clinical trials fundamental conclusions. Pharmacological interventions involving nicotine replacement and antidepressant use, with bupropion as the first line agent, and psychossocial interventions involving cognitive behavioral therapies and face-to-face or phone-based motivational interventions are proving to be efficacious. Pharmacotherapies increase two-fold the chance of abstinence and face-to-face or phonebased psychosocial interventions increase the chance of quitting smoking in 1.5 to 2.5 times in comparison to individuals who try to quit smoking by themselves. These data support health professionals to decide the most effective treatment for individual smokers, according to the available interventions tested, their efficacy and the benefits of use.
\end{abstract}

Keywords: tobacco; smoking; therapy; drug therapy

$\mathrm{O}$ consumo de tabaco está associado a doenças cardiovasculares, doenças pulmonares obstrutivas crônicas, câncer de pulmão e outras neoplasias (Doll, Peto, Boreham, \& Sutherland, 2004; Ezzati, Lopez, Rodgers, Vander, \& Murray, 2002). A mortalidade geral é duas vezes maior nos fumantes, quando comparada aos não-fumantes. Além disso, o tabaco é uma das principais causas evitáveis de mortes prematuras (Ezzati, Lopez, Rodgers, Vander, \& Murray, 2002).

A cessação do tabagismo traz consideráveis benefícios à 
saúde, pois indivíduos que param de fumar evitam a maioria das doenças associadas ao tabaco (Peto et al., 2000). Apesar de todo o conhecimento científico acumulado sobre os riscos do tabaco, as tendências do seu consumo ainda são alarmantes. Um terço da população mundial, com 15 anos ou mais, é fumante. No Brasil, 9\% da população são dependentes de tabaco. Sendo que $16,4 \%$ desejam parar ou reduzir seu consumo (Carlini, Galduróz, Noto, \& Napo, 2002).

Intervenções farmacológicas e comportamentais têm sido propostas na tentativa de auxiliar indivíduos a pararem de fumar (Fiore, 2000). Embora 80\% dos fumantes desejem parar de fumar, apenas 3\% conseguem fazê-lo por si mesmos e, somente $7 \%$ dos que tentam parar sozinhos se mantêm abstinentes por um período longo de tempo. A taxa de sucesso de abstinência pode aumentar em $15 \%$ a $30 \%$ com a utilização de intervenções psicossociais e farmacológicas (Cinciripini, Hecht, Henningfield, Manley, \& Kramer, 1997).

Este artigo tem como objetivo abordar as características fundamentais das intervenções farmacológicas e psicossociais e sua eficácia para o tratamento dos dependentes de tabaco. A proposta desta revisão foi a de apresentar informações baseadas na eficácia, ou seja, na demonstração de uma diminuição significativa de tabagistas, favorecendo o desfecho de cessação de uso de tabaco, por ao menos seis meses, no grupo de pacientes submetidos à intervenção em teste, em comparação com outra intervenção controle. Para tanto, utilizaram-se ensaios clínico-controlados ou meta-análises, que são os estudos que demonstram a recomendação A e são, portanto, os que melhor apontam as evidências científicas para apoiar a utilização de uma determinada intervenção farmacológica ou psicossocial. Nas meta-análises são associados os resultados de vários estudos clínicos independentes, mostrando-se mais propensos a serem reais e objetivos, e levando a uma estimativa mais precisa do efeito. Nestas, depois de extraídos os dados relevantes de cada estudo, é possível calcular uma estimativa geral relacionada ao desfecho após uma intervenção, como, por exemplo, a razão de chances sumário (OR) e seu intervalo de confiança (IC). O efeito sumário é um efeito médio, ponderado de acordo com o tamanho de cada estudo. Os estudos clinico-controlados e as meta-análises são estudos com grau de recomendação A, ou seja, aqueles estudos cujos resultados são irrefutáveis do ponto de vista metodológico e nos quais os profissionais podem basear-se para a escolha do melhor tratamento (Sackett, Straus, Richardson, Rosenberg, \& Haynes, 2003). Nesta revisão foram utilizadas as bases de referências bibliográficas PUBMED e SciELO, aplicando-se os descritores tobacco, tobacco use disorder, ou smoking cessation; e os limites relacionados a meta-analysis, practice guideline, e randomized controlled trial.

\section{Intervenções farmacológicas}

Interessados que estão tentando parar de fumar podem fazer uso de terapias farmacológicas exceto em situações especiais como o uso de menos de 10 cigarros por dia, gestantes, adolescentes e contra indicações-médicas (Fiore, 2000; Focchi \& Braun, 2005). O tratamento farmacológico para dependência de nicotina inclui diferentes métodos. As doses são administradas conforme a necessidade de cada fumante, considerando-se o grau de dependência, a tolerância e a preferência do indivíduo (Woerpel, Wright, \& Wetter, 2006). Os fármacos utilizados classificam-se conforme o mecanismo de ação e a eficácia. Destacam-se os agentes nicotínicos (terapia de reposição de nicotina-TRN) e os não-nicotínicos (antidepressivos) (Viegas \& Reichert, 2004). A vareniclina é um fármaco promissor que atua no mecanismo neurobiológico da dependência (Jorenby et al., 2006). A TRN e a bupropiona são de primeira linha e as demais intervenções, como a nortriptilina e a clonidina, são de segunda linha (Fiore, 2000; Focchi \& Braun, 2005; Marques et al., 2001). $\mathrm{Na}$ Tabela 1, estão resumidas as intervenções farmacológicas, com suas razões de chances de sucesso de abstinência.

A terapia de reposição de nicotina (TRN) refere-se à administração de nicotina para substituir o uso do tabaco (Mitrouska, Bouloukaki, \& Siafakas, 2006). A eficácia da TRN

Tabela 1

Resumo de meta-análises da eficácia de intervenções farma cológicas para cessação do tabagismo

\begin{tabular}{|c|c|c|c|c|}
\hline $\begin{array}{l}\text { Intervenções } \\
\text { farmacológicas }\end{array}$ & Descrição & $\begin{array}{c}\text { Estimativa do efeito } \\
\text { Razão ch ances } \\
\text { (IC 95\%) }\end{array}$ & $\begin{array}{l}\text { Número de } \\
\text { estudos }\end{array}$ & Custo/dia \\
\hline $\begin{array}{l}\text { Te rapia de reposição de } \\
\text { nicotina (TRN) } \\
\text { (Silagy et al., 2004) }\end{array}$ & $\begin{array}{l}\text { TRN } \\
\text { Vs } \\
\text { Controle }\end{array}$ & $\begin{array}{c}1,77 \\
(1,66-1,88)\end{array}$ & 103 & $\begin{array}{l}\text { Goma } 2 \mathrm{mg}: \\
\mathrm{R} \$ 11,75 \\
\text { Adesivo } 14 \mathrm{mg} \text { : } \\
\mathrm{R} \$ 5,95\end{array}$ \\
\hline $\begin{array}{l}\text { Bupropiona } \\
\text { (Hughes et al., 2004) }\end{array}$ & $\begin{array}{l}\text { Bupropiona } \\
\text { Vs } \\
\text { Controle }\end{array}$ & $\begin{array}{c}2,06 \\
(1,77-2,40)\end{array}$ & 19 & $\begin{array}{l}\text { 300mg: } \\
\mathrm{R} \$ 3,53\end{array}$ \\
\hline $\begin{array}{l}\text { Nortriptilina } \\
\text { (Hughes et al., 2004) }\end{array}$ & $\begin{array}{l}\text { Nortriptilina } \\
\text { Vs } \\
\text { Controle }\end{array}$ & $\begin{array}{c}2,14 \\
(1,49-3,06)\end{array}$ & 4 & $\begin{array}{l}75 \mathrm{mg}: \\
\mathrm{R} \$ 0,47\end{array}$ \\
\hline $\begin{array}{l}\text { Clonidina } \\
\text { (Gourlay et al., 2004) }\end{array}$ & $\begin{array}{l}\text { Clonidina } \\
\text { Vs } \\
\text { Controle }\end{array}$ & $\begin{array}{c}1,89 \\
(1, .30-2,74)\end{array}$ & 6 & $\begin{array}{l}0,15 \mathrm{mg}: \\
\mathrm{R} \$ 0,16\end{array}$ \\
\hline
\end{tabular}


está relacionada à reposição de nicotina de modo mais lento e com menores picos que o tabaco, além de proporcionar, em alguns casos, estimulação oral e rituais de uso (Focchi \& Braum, 2005).

A TRN diminui os sintomas da síndrome de abstinência, reduzindo os efeitos reforçadores negativos da falta de nicotina (Henningfield, Fant, Buchhalter, \& Stitzer, 2005; Marques \& Ribeiro, 2003). No Brasil, as formas disponíveis incluem a goma de mascar e os sistemas transdérmicos. A goma de mascar contém $2 \mathrm{mg}$ de nicotina, a qual é liberada e absorvida rapidamente na mucosa oral. A média de consumo é de 10 gomas/dia, podendo chegar até 20 gomas (Balbani \& Montovani, 2005; Marques \& Ribeiro, 2003). Os adesivos transdérmicos são encontrados nas versões de 7, 14 e $21 \mathrm{mg}$ por unidade. A nicotina é liberada de forma gradual dos adesivos durante todo o dia. O efeito adverso mais comum é a irritação na pele (Lancaster, Stead, Silagy, \& Sowden, 2000). A reposição com adesivo é indicada por um período médio de 8 semanas e deve-se aplicar um adesivo por dia (Viegas \& Reichert, 2004). Nos Estados Unidos, além destes produtos, há preparação de nicotina em spray nasal, inalador, aerossol, pastilhas sublinguais e comprimidos (Balbani \& Montovani, 2005).

A TRN pode ser aplicada em tabagistas que consomem mais de 10 cigarros por dia (Marques \& Ribeiro, 2003; Silagy, Lancaster, Stead, Mant, \& Fowler, 2004). Em dependentes pesados, inicia-se o tratamento com $21 \mathrm{mg}$, podendo diminuir a dose semanalmente. Para os demais tabagistas, a dose inicial preconizada é de $15 \mathrm{mg}$ de nicotina por dia. As doses devem ser reduzidas ou suspensas após 2 a 3 meses, e a duração do tratamento não deve ultrapassar 6 meses. (Marques \& Ribeiro, 2003; Viegas \& Reichert, 2004). A TRN, quando comparada com grupo controle, aumenta a chance de cessação do consumo de tabaco $(\mathrm{OR}=1,77 ; \mathrm{IC} 95 \%$ : 1,66 a 1,88). Porém, é um tratamento com custo elevado, o qual pode ser utilizado por uma pequena parcela da população (Tabela 1).

Os antidepressivos têm sido utilizados, com a expectativa de melhorar os sintomas depressivos da síndrome de abstinência (Hughes, 2003; Woerpel et al., 2006). A bupropiona é um antidepressivo que inibe a recaptação de noradrenalina e dopamina nas sinapses. O pico de concentração plasmática é atingido em 3 horas, e sua meia-vida é de 19 horas. A metabolização é hepática e a excreção, renal. Supõe-se que o mecanismo de ação da bupropiona esteja relacionado com uma redução no transporte neural de dopamina e noradrenalina ou com antagonismo nos receptores nicotínicos, desencadeando uma redução na compulsão pelo uso de cigarros (Focchi \& Braun, 2005). A bupropiona diminui sintomas da síndrome de abstinência, como a depressão (Henningfield et al., 2005). A dose preconizada é de $150 \mathrm{mg}$ por 3 dias e, a seguir, 300mg/dia por 7 a 12 semanas (Marques et al., 2001). Há indicação para indivíduos que fumam de 10 a 15 cigarros por dia e que estão motivados a parar (Coleman, 2001). Boca seca e insônia são os efeitos mais comuns associados ao seu uso. Existe um pequeno risco de convulsão, nos indivíduos com histórico de convulsões ou pré-disposição às crises (Henningfield, et al., 2005). Quando comparada com a TRN, a bupropiona apresenta maior chance de o indivíduo alcançar a abstinência (Tabela 1).
A nortriptilina bloqueia a recaptação de noradrenalina na pré-sinapse, aumentando sua concentração na fenda sináptica. A meia-vida plasmática varia de $12 \mathrm{~h}$ a $56 \mathrm{~h}$, sendo metabolizada pelo fígado e excretada pelos rins. Os principais efeitos adversos são: boca seca, tremores, visão turva, sedação, retenção urinária, leves dores de cabeça, mãos trêmulas, risco de arritmia (Scharf \& Shiffman, 2004), taquicardia, ganho ou perda de peso e hipotensão baixa (Focchi \& Braun, 2005; Henningfield et al., 2005; Scharf \& Shiffman, 2004). Apesar desses efeitos, a nortriptilina pode ser vantajosa como tratamento alternativo do tabagismo, pois tem menor efeito colinérgico se comparada a outros tricíclicos, e menor risco de provocar convulsões (Focchi \& Braun, 2005). O uso de nortriptilina é indicado quando não é possível prescrever bupropiona ou TRN. O tratamento deve ser iniciado 2 a 4 semanas antes da retirada do cigarro, com doses progressivas de $25 \mathrm{mg}$ por dia até $75 \mathrm{mg}$, com tempo de uso variável (Marques \& Ribeiro, 2003; Viegas \& Reichert, 2004). A utilização desse fármaco no processo de cessação do tabagismo aumenta a chance de abstinência com eficácia semelhante ao da bupropiona. É um tratamento menos oneroso e mais eficaz do que a TRN e a bupropiona isoladas (Tabela 1). Apresenta como vantagem o fato de estar disponível para a população através do Sistema Único de Saúde (SUS).

A clonidina, agonista alfa-2 noradrenérgico, tem sido utilizado tanto para o tratamento de hipertensão quanto para cessação do tabagismo, sendo tratamento de segunda linha por induzir constipação, boca seca, cansaço e irritação cutânea (Mitrouska et al., 2006; Viegas \& Reichert, 2004). Este fármaco pode auxiliar fumantes que apresentam agitação e ansiedade quando param de fumar (Gourlay, Stead, \& Benowitz, 2004). Sua administração deve iniciada alguns dias antes da cessação. A dose inicial é de $0,05 \mathrm{mg}$ até atingir $0,15 \mathrm{mg}$ (Viegas \& Reichert, 2004). É um fármaco com eficácia similar e com custo inferior a TRN, porém, em decorrência de seus efeitos adversos sua prescrição é limitada (Tabela 1).

O primeiro tratamento específico para a cessação do tabagismo que age no mecanismo neurobiológico de dependência à nicotina é a vareniclina (Foulds, 2006). Este fármaco ligase seletivamente aos receptores nicotínicos da acetilcolina. Quando se liga, a vareniclina atua como um agonista parcial, que ajuda a diminuir os sintomas de abstinência. Desta forma, reduz os efeitos de reforço positivo da nicotina (Henningfield et al., 2005). A vareniclina tem eficácia e segurança (Jorenby et al., 2006), além disso, é mais eficaz que placebo e bupropiona num tratamento de 24 semanas (OR=3,09; CI 95\%: 1,95 a 4,91) (Gonzales et al., 2006). A vareniclina já foi aprovada pela Agência Nacional de Vigilância Sanitária, e foi disponibilizada no Brasil no segundo semestre de 2007 (Agência Nacional de Vigilância Sanitária, 2006).

\section{Intervenções psicossociais}

Os diferentes modelos de tratamento variam de acordo com a base teórica e as técnicas utilizadas. As intervenções podem ser diferenciadas pela intensidade (freqüência ou duração do tratamento), pelo setting que é utilizado (ambulatorial ou hospitalar), e pela forma em que é oferecida (individual, grupo, ou por meio de outro canal de comunicação como, por exemplo, 
o telefone) (Baker \& Lee, 2003). A maioria das intervenções psicossociais para dependência química compartilha de técnicas como a psicoeducação, reforço da auto-eficácia, suporte e encorajamento da relação terapêutica (Shearer, 2007). As intervenções podem ser caracterizadas como breves ou intensivas. As breves incluem material de auto-ajuda e aconselhamento face a face ou telefônico. As intervenções intensivas são geralmente oferecidas por profissionais especializados e têm formato individual ou em grupo (Lancaster \& Stead, 2005).

$\mathrm{Na}$ Tabela 2, estão resumidas algumas intervenções psicossociais com as suas razões de chances de sucesso de parada.

Tabela 2.

Resumo de meta-análises da eficácia de intervenções psicossociais para cessação do tabagismo

\begin{tabular}{|c|c|c|c|}
\hline $\begin{array}{l}\text { Intervenções } \\
\text { psicos sociais }\end{array}$ & Descrição & $\begin{array}{l}\text { Estimativa do efeito } \\
\text { Razão ch ance s } \\
\text { (IC 95\%) }\end{array}$ & $\begin{array}{l}\text { Número } \\
\text { de } \\
\text { estudos }\end{array}$ \\
\hline $\begin{array}{l}\text { Aconselhamento breve de um } \\
\text { médico } \\
\text { (Lan caster \& St ead, 2004) }\end{array}$ & $\begin{array}{l}\text { Aconselhamento breve } \\
\text { Vs } \\
\text { Não aco nselhamento }\end{array}$ & $\begin{array}{c}1,74 \\
(1,48-2,05)\end{array}$ & 17 \\
\hline $\begin{array}{l}\text { Aconselhamento } \\
\text { intensivo de um médico } \\
\text { (Lancaster \& St ead, 2004) }\end{array}$ & $\begin{array}{l}\text { Aconselhamento } \\
\text { intensivo } \\
\text { Vs } \\
\text { Aconselhamento } \\
\text { mínimo }\end{array}$ & $\begin{array}{c}1,44 \\
(1,24-1,67)\end{array}$ & 15 \\
\hline $\begin{array}{l}\text { Aconselhamento } \\
\text { telefônico } \\
\text { (Stead, La ncaster, \& Perera, } \\
\text { 2002) }\end{array}$ & $\begin{array}{l}\text { Aconselhamento } \\
\text { telefônico } \\
\text { Vs } \\
\text { Controle }\end{array}$ & $\begin{array}{c}1,56 \\
(1,38-1,77)\end{array}$ & 27 \\
\hline $\begin{array}{l}\text { Materiais de } \\
\text { auto-ajuda } \\
\text { (Lancaster \& St ead, 2005) }\end{array}$ & $\begin{array}{l}\text { Material de auto-ajuda } \\
\text { Vs } \\
\text { Controle }\end{array}$ & $\begin{array}{c}1,24 \\
(1,07-1,45)\end{array}$ & 11 \\
\hline \multirow[t]{2}{*}{$\begin{array}{l}\text { Te rapia de Grupo } \\
\text { (Lan caster \& St ead, 2005) }\end{array}$} & $\begin{array}{l}\text { Te rapia de grupo } \\
\text { Vs } \\
\text { Material de auto-ajuda }\end{array}$ & $\begin{array}{c}2,64 \\
(1,89-3,69)\end{array}$ & 13 \\
\hline & $\begin{array}{l}\text { Te rapia de grupo } \\
\text { Vs } \\
\text { Te rapia individual }\end{array}$ & $\begin{array}{c}0,86 \\
(0,66-1,12)\end{array}$ & 5 \\
\hline
\end{tabular}

A entrevista motivacional é uma das intervenções psicossociais que vem sendo utilizada para cessação de comportamentos dependentes. Está baseada em três conceitos importantes: a motivação, a qual é definida como a probabilidade de que uma pessoa inicie, dê continuidade e permaneça num processo de mudança específico (Miller, 1985); a ambivalência, experiência de conflito psicológico para escolher entre dois caminhos; e a prontidão para mudança que está baseada no Modelo Transteórico (Prochaska \& DiClemente, 1982). Este modelo preconiza que a mudança comportamental é um processo e que as pessoas têm diversos níveis de motivação para mudar comportamentos. Com isso, permite planejar intervenções e programas que atendam às necessidades particulares dos indivíduos (Prochaska \& DiClemente, 1984).

O modelo transteórico descreve 5 estágios motivacionais, pelos quais os indivíduos passam, quando modificam um comportamento problema. O primeiro, a Pré-Contemplação, o indivíduo não demonstra consciência suficiente de que tem problemas. Quando a pessoa passa a considerar seu comportamento como um problema, com a possibilidade de mudanças, ela entra no estágio de Contemplação. Seguindo para o estágio de Preparação, a pessoa começa a construir tentativas para mudar seu comportamento. Quando estas tentativas são colocadas em prática, a pessoa encontra-se no estágio de $A c ̧ a ̃ o$, ocorrendo uma implementação de planos para a modificação do comportamento problema. Finalmente, o sucesso da mudança ocorre no estágio de Manutenção, onde a pessoa modifica seu estilo de vida, evitando a recaída, atingindo abstinência e consolidando as mudanças. A recaída pode estar presente, obrigando novamente o dependente a passar várias vezes pelos estágios de mudança, antes de atingir a manutenção em longo prazo (DiClemente et al., 1991).

Poucos fumantes $(10 \%)$ encontram-se prontos para modificarem seu hábito de fumar. Portanto, as intervenções devem envolver a avaliação do estágio motivacional e a aplicação de técnicas adequadas a cada estágio (Hughes, 2003). A entrevista motivacional é uma ferramenta bastante útil em várias fases do tratamento e especialmente importante em casos nos 
quais os clientes estejam ambivalentes e resistentes à mudança. Além disso, por tratar-se de uma técnica breve e diretiva, possui indicação para atender vários settings como o ambulatório, o hospital e mais recentemente vem sendo disponibilizada através de tele-consulta. Vários estudos relatam à utilização da entrevista motivacional por telefone para atendimento de tabagistas (Borland, Balmford, Segan, \& Livingston, 2003; Curry, McBride, Grothaus, Louis, \& Wagner, 1995; Ludman, Curry, Meyer, \& Taplin, 1999; Mazoni, Bish, Freese, Ferigolo \& Barros, 2007; Zhu, Tedeschi, Anderson, \& Pirce, 1996; Zhu, et al., 2002). Por outro lado quando telefonam para um serviço de tele atendimento, $83 \%$ encontram-se no estágio de ação (Mazoni, 2007).

A terapia cognitivo-comportamental tem como finalidade informar o tabagista sobre os riscos do cigarro e os benefícios de parar de fumar. Além disso, possibilita apoiar o cliente durante o processo de cessação, oferecendo orientações para que possa lidar com a síndrome de abstinência, a dependência psicológica e os comportamentos associados ao comportamento de fumar. No tratamento cognitivo-comportamental do tabagismo utilizam-se técnicas de treinamento de habilidades, de solução de problemas, bem como o apoio social (Fiore, 2000). O aconselhamento tem formato individual ou em grupo e deve ser conduzido por profissionais especializados e treinados para esse propósito (Fiore, 2000; West, 2004). O programa envolve múltiplos contatos por um período mínimo de 4 semanas (West, 2004). Alguns programas incluem materiais de auto-ajuda como folhetos, vídeos ou fitas de áudio (Lancaster \& Stead, 2005).

Não há evidências para afirmar se uma das modalidades, em grupo ou individual, é mais eficaz (Stead \& Lancaster, 2005) (Tabela 2). O tratamento em grupo oportuniza maior suporte social e maior facilitação da discussão de situações de risco, bem como das estratégias para lidar com as mesmas. Por outro lado, o tratamento individual permite atenção e adaptação às características específicas de cada paciente. A escolha deve ser baseada na preferência do paciente (Presman, Carneiro, \& Gigliotti, 2005). A terapia cognitivo-comportamental em grupo é uma abordagem que demonstra eficácia, aumentando a chance de abstinência prolongada (Tabela 2).

A intervenção breve geralmente é aplicada por profissionais especializados, quando o usuário de drogas busca auxílio para mudança de seu comportamento problema. Em nível de atenção primária à saúde, profissionais não especializados podem oferecer intervenção a tabagistas que, por outros motivos procuram atendimento de saúde (Heather, 1996; Roche \& Freeman, 2004). O objetivo principal é identificar e aconselhar tabagistas, aumentando a motivação interna (Severson \& Hatsukami, 1999). Essa técnica é composta por um conjunto de estratégias ou procedimentos que variam quanto à duração, estrutura, metas, pessoal responsável, meio de comunicação, ambiente de execução e também em relação aos seus diferentes fundamentos teóricos (Marques \& Furtado, 2004). Muitos dos dados de pesquisa focaram-se sobre as intervenções breves, sem definir claramente os diferentes métodos e estratégias utilizadas, pois há diferença em um aconselhamento breve e intervenções breves motivacionais (Butler et al., 1999). A intervenção breve motivacional integra diferentes estratégias para aumentar a motivação para mudança de comportamentos. É uma forma simplificada da entrevista motivacional desenvolvida para ser utilizada nos cuidados de atenção primária a saúde (Rollnick, Heather, \& Bell 1992). Esse método tem como vantagens: curta duração, realizada em uma única sessão, com variação entre 5 e 60 minutos de aconselhamento; pode ser utilizadas por não especialistas (Miller \& Rollnick, 2001; Rollnick, Heather, \& Bell, 1992).

Miller e Rollnick (2001), enumeraram seis elementos indispensáveis para trabalhar estratégias de mudança. Estes elementos podem ser sumarizados pelo acrônimo inglês FRAMES: Feedback (devolução), que é o uso da informação com base nos resultados obtidos na avaliação inicial estruturada e objetiva da real situação do cliente; Responsibility (responsabilidade) é a ênfase na responsabilidade pessoal do cliente e na liberdade de escolha; Advice (recomendação) é proporcionar ao cliente conselhos claros e diretos, sobre a necessidade de mudança e como ela pode ser obtida; Menu (inventário) é oferecer opções de escolha para o cliente, mostrando alternativas para mudança; Empathy (empatia) é a aceitação do cliente, entendendo seu processo sem julgá-lo e escutando-o de forma reflexiva; e Selfefficacy (auto-eficácia) que reforça a crença do cliente na própria capacidade de mudança.

A intervenção breve motivacional produz melhores resultados do que um simples aconselhamento, especialmente entre aqueles que não estão prontos para parar de fumar (Butler et al., 1999), dobra as taxas de cessação, gasta menos tempo no atendimento dos pacientes, previne doenças crônicas relacionadas ao cigarro e tem alto impacto custo/benefício (Roche \& Freeman, 2004). Esta intervenção também tem sido oferecida por meio de ligações telefônicas (Ludman et al., 1999; Mazoni et al., 2007; Ramelson, Friedman, \& Ockene, 1999; Zhu et al., 1996) e materiais de auto-ajuda (Britt, Hudson, Blampied, \& 2004).

O aconselhamento telefônico para tabagistas tem sido utilizado em diversas populações em associação com a farmacoterapia (Boyle et al., 2005), em fumantes que estão aguardando procedimentos cirúrgicos (Bottorff et al., 2004), para os pais fumantes (Abdullah, Mak, Loke, \& Lam, 2005), gestantes (Solomon \& Flynn 2005) ou fumantes da população em geral (Helgason et al., 2004). Linhas telefônicas (helplines, quitlines, crisis lines) são amplamente utilizadas na América do Norte e Europa (Bentz et al., 2006; Gilbert, Sutton, \& Sutherland, 2005; Gilbert \& Sutton, 2006) e mais recentemente no Brasil (Mazoni et al., 2006) oferecendo aconselhamento breve e apoio a fumantes que desejam parar de fumar sozinhos ou com intervenção mínima (Gilbert et al., 2005; Wakefield \& Borland, 2000). É um método que apresenta vantagens, não somente pelo fato de que atende um grande número de indivíduos, mas também porque favorece àqueles tabagistas que não têm acesso (ou interesse por) a outros tratamentos (Gilbert \& Sutton, 2006; Paul et al., 2004; Wakefield \& Borland, 2000; Zhu et al., 1996).

Os serviços telefônicos para tabagistas são realizados por meio do aconselhamento telefônico reativo ou proativo. No aconselhamento telefônico proativo, as ligações para o cliente são iniciadas pelo serviço. O consultor do serviço entra em contato com o cliente, a partir de uma primeira ligação reativa 
ou por meio de um cadastro de clientes que realizaram consultas com profissionais da saúde (El-Bastawissi et al., 2003).

$\mathrm{O}$ aconselhamento reativo tem como característica a disponibilização de campanhas de divulgação na mídia para alcançar a população desejada (Lichtenstein, Glasgow, Lando, Ossip-Klein, \& Boles, 1996). Esse aconselhamento pode ser individualizado ou por meio da comunicação com um sistema computadorizado, o qual repete mensagens gravadas aos que ligam para o serviço (Borland et al., 2003; Friedman, Stollerman, Mahoney, \& Rozenblyum, 1997). Devido à dificuldade de avaliação da efetividade de linhas telefônicas reativas poucas pesquisas têm sido conduzidas (Lichtenstein et al., 1996; Presman et al., 2005). Em nosso país, um ensaio clínico controlado randomizado apontou que o aconselhamento telefônico reativo baseado na intervenção breve motivacional, aumenta a chance de abstinência por seis meses $(\mathrm{OR}=1,95$; IC 95\%: 1,5-1,1) e reduz o número de cigarros fumados por dia (OR = 2,3; IC 95\%: 1,6-3,2; Mazoni, 2007).

$\mathrm{O}$ aconselhamento telefônico tem potencial para complementar as intervenções face a face, substituí-las ou fortalecer as intervenções de auto-ajuda. Esse tipo de aconselhamento pode ser útil para planejar a parada e auxiliar os fumantes na prevenção da recaída durante o período inicial de abstinência (Mazoni et al., 2006). Ainda que intervenções intensivas face a face demonstrem sucesso para cessação do tabagismo existem dificuldades para disponibilizá-las em larga escala à população, pelo custo, pela dificuldade de horários e de deslocamento (Stead \& Lancaster, 2005). De uma forma geral, pode-se dizer que o aconselhamento telefônico aumenta as taxas de cessação do tabagismo e o número de fumantes fazendo tentativas de abstinência (Klein \& Mcintosh, 2003; Miller, Wakefield, \& Roberts, 2003), pois atinge a população em larga escala. Desta forma, pode diminuir a prevalência do tabagismo, sendo uma medida útil em saúde pública (Klein \& Mcintosh, 2003; Miller et al., 2003).

Material de auto-ajuda também objetiva aumentar a motivação para mudança e informar sobre como deixar de fumar (Presman et al., 2005). Intervenções comportamentais podem ser disponibilizadas por meio desses materiais, incluindo os folhetos e materiais escritos, fitas de áudio, fitas de vídeo e programas de computador (Lancaster et al., 2000). Os materiais abordam conseqüências sociais do hábito de fumar, problemas de saúde decorrentes do uso, estratégias específicas para auxiliar o abandono do tabaco e informações sobre a síndrome de abstinência e a recaída (Balanda, Lowe, \& Fleming, 1999). Os materiais de auto-ajuda também prestam suporte de baixo custo (Borland, Balmford, \& Hunt 2004) e atingem um número maior de pessoas quando comparados à intervenção face a face (Lancaster et al., 2000), o que poderá resultar num número maior de tabagistas abstinentes (Balanda et al., 1999). Por outro lado, em populações pouco alfabetizadas, não pode ser empregado. Os manuais personalizados são mais efetivos quando comparados aos padronizados (Lancaster \& Stead, 2005).

\section{Considerações finais}

Significantes avanços na farmacoterapia para cessação do tabagismo resultaram num melhor entendimento dos mecanismos de ação da nicotina e no surgimento de novos fármacos que atuam no mecanismo neurobiológico da dependência. A farmacoterapia é uma boa alternativa para reduzir os sintomas de abstinência, melhorando as taxas de cessação, e a integração de intervenções comportamentais aumenta ainda mais a chance de sucesso do tratamento e podem ser úteis em todas as fases do tratamento da dependência de nicotina (Foulds, 2006).

Muitos tabagistas em nosso país desejam parar de fumar ou reduzir o consumo de tabaco (Carlini et al., 2002). Atualmente, há facilidade para os profissionais de saúde decidirem qual o melhor tratamento para o paciente tabagista, já que o avanço da ciência permite uma prática cada vez mais baseada em informações fundamentadas em evidência. Tabagistas que utilizaram intervenções eficazes aumentaram as chances de sucesso de abstinência, quando comparados com a não utilização de terapias, ou placebo (Fiore, 2000). Efeitos positivos também foram encontrados quando o paciente foi informado das intervenções disponíveis, sua eficácia e o benefício de sua utilização (Willemsen, Wiebing, van Emst, \& Zeeman, 2006).

Dessa forma, faz-se necessário motivar os tabagistas para mudança do seu comportamento aditivo, conscientizando-os sobre o potencial benefício do tratamento e disponibilizar profissionais capacitados para indicar o tratamento mais eficaz de forma personalizada. Esta revisão apresenta as melhores evidências publicadas até o momento, obtidas através de estudos de meta-análises, para apoiar a utilização de algumas medidas farmacológicas e psicossociais, para auxiliar no processo de abstinência de pacientes tabagistas.

\section{Agradecimentos}

As autoras agradecem o apoio de: SENAD, AAPEFATO, CAPES (Cláudia G. Mazoni) e CNPQ (Helena M. T. Barros, Pesquisador 1C).

\section{Referências}

Abdullah, A. S., Mak, Y. W., Loke, A. Y., \& Lam, T. H. (2005). Smoking cessation intervention in parents of young children: a randomized controlled trial. Addiction, 100, 1731-1740

Agência Nacional de Vigilância Sanitária (2006). Legislação em vigilância sanitária: vareniclina. Obtido em 20 de outubro de 2006, de http://www. anvisa.gov.br.

Baker, A., \& Lee, N. K. (2003). A review of psychosocial interventions for amphetamine use. Drug and Alcohol Review, 22, 323-235.

Balanda, K. P., Lowe, J. B., \& Fleming, M. L. O. (1999). Comparison of two self-help smoking cessation booklets. Tobacco Control, 8, 57-61.

Balbani, A. P. S., \& Montovani, J. C. (2005). Métodos para abandono do tabagismo e tratamento da dependência da nicotina. Revista Brasileira Otorrinolaringologia, 71, 820-827.

Bentz, C. J., Bayley, K. B., Bonin, K. E., Fleming, L., Hollis, J. F., \& McAfee, T. (2006). The feasibility of connecting physician offices to a state-level tobacco quitline. American Journal of Preventive Medicine, 30, 31-37.

Borland, R., Balmford, J. \& Hunt, D. (2004). The effectiveness of personally tailored computer-generated advice letters for smoking cessation. Addiction, 999, 369-377. 
Borland, R., Balmford, J., Segan, V., \& Livingston, P. (2003). The effectiveness of personalized smoking cessation strategies for callers to a quitline service. Addiction, 98, 837-846.

Bottorff, J. L., Johnson, J. L., Moffat, B., Fofonoff, D., Budz, B., \& Groening, M. (2004). Synchronizing clinician engagement and client motivation in telephone counseling. Qualitative Health Research, 14, 462-477.

Boyle, R. G., Solberg, L. I., Asche, S. E., Boucher, J. L., Pronk, N. P., \& Jensen C. J. (2005). Offering telephone counseling to smokers using pharmacotherapy. Nicotine \& Tobacco Research, 7, 19-27.

Britt, E., Hudson, S. M., \& Blampied, N. M. (2004). Motivational interviewing in health settings: a review. Patient Education Counseling, 53, 147-155.

Butler, C., Rollnick, S., Cohen, D., Bachman, M., Russell, I., \& Stott N. (1999). Motivational consulting versus brief advice for smoker in general practice: a randomized trial. British Journal of General Practice, 49, 611-616.

Carlini, E. A., Galduróz, J. C. F., Noto, A. R., \& Napo, S. A. (2002). I Levantamento domiciliar sobre o uso de drogas psicotrópicas: estudo envolvendo as 107 maiores cidades do país. São Paulo: CEBRID.

Cinciripini, P. M., Hecht, S. S., Henningfield, J. E., Manley, M. W., \& Kramer, B. S. (1997). Tobacco addiction: implications for treatment and cancer prevention. Journal of the National Cancer Institute, 89, 1852-1867.

Coleman, T. (2001). Smoking cessation: integrating recent advances into clinical practice. Thorax, 56, 579-582.

Curry, S. J., McBride, C., Grothaus, L. C., Louie, D., \& Wagner, E. H. (1995). A randomized trial of self-help materials, personalized feedback, and telephone counseling with nonvolunteer smokers. Journal of Consulting and Clinical Psychology, 63, 1005-1014.

DiClemente, C. C., Prochaska, J. O., Fairhurst, S. K., Velicer, W. F., Velásquez, M. M., \& Rossi, J. S. (1991). The process of smoking cessation: an analysis of precontemplation, contemplation, and preparation stages of change. Journal of Consulting and Clinical Psycholoy, 59, 295-304.

Doll, R., Peto, R., Boreham, J., \& Sutherland, I. (2004). Mortality in relation to smoking: 50 years' observations on male British doctors. British Medical Journal, 328, 1507-1519.

El-Bastawissi, A., McAfee, T., Zbikowski, S. M., Hollis, J., Stark, M., Wassum, K., et al. (2003). The uninsured and medicaid Oregon tobacco user experience in a real world, phone based cessation programme. Tobacco Control, 12, 45-51.

Ezzati, M., Lopez, A. D., Rodgers, A., Vander Hoorn, S., \& Murray, C. J. (2002). Comparative risk assessment collaborating group; selected major risk factors and global and regional burden of disease. Lancet, 360, 1347-1360.

Fiore, M. C. (2000). Treating tobacco use and dependence: an introduction to the US public health service clinical practice guideline. Respiratory Care, 45, 1196-1199.

Focchi, G. R. A., \& Braun, I. M. (2005). Tratamento farmacológico do tabagismo. Revista de Psiquiatria Clínica, 32, 259-266.

Foulds, J. (2006). The neurobiological basis for partial agonist treatment of nicotine dependence varenicline. International Journal of Clinical Practice, 60, 571-576.

Friedman, R. H., Stollerman, J. E., Mahoney, D. M., \& Rozenblyum, L. (1997). The virtual visit: using telecommunications technology to take care of patients. Journal of the American Medical Informatics Association, 4, 413-425.

Gilbert, H., \& Sutton, S. (2006). Evaluating the effectiveness of proactive telephone counseling for smoking cessation in a randomized controlled trial. Addiction, 101, 590-598.

Gilbert, H., Sutton, S., \& Sutherland, G. (2005). Who calls quit? The characteristics of smokers seeking advice via a telephone helpline compared with smokers attending a clinic and those in the general population. Public Health, 119, 933-939.

Gonzales, D., Rennard, S. I., Nides, M., Oncken, C., Azoulay, S., Billing, C. B., et al. (2006). Varenicline, an alpha4beta2 nicotinic acetylcholine receptor partial agonist, vs sustained-release bupropion and placebo for smoking cessation: a randomized controlled trial. Journal of the American Medical Association, 5, 47-55.

Gourlay, S. G., Stead, L. F., \& Benowitz, N. L. (2004). Clonidine for smoking cessation. Cochrane Database of Systematic Reviews, CD000058.

Heather, N. (1996). The public health and brief interventions for excessive alcohol consumption: the British experience. Addictive Behaviors, 21, 857-868.

Helgason, A. R., Tomson, T., Lund, K. E., Galanti, R., Ahnve, S., \& Gilljam, H. (2004). Factors related to abstinence in a telephone helpline for smoking cessation. European Journal of Public Health, 14, 306-310.

Henningfield, J. E., Fant, R. V., Buchhalter, A. R., \& Stitzer, M. L. (2005) Pharmacotherapy for nicotine dependence. A Cancer Journal for Clinicians, 55, 281-299.

Hugues, J. R. (2003). Motivating and helping smokers to stop smoking. Journal of General Internal Medicine, 18, 1053-1057.

Jorenby, D. E., Hays, J. T., Rigotti, N. A., Azoulay, S., Watsky, E. J., Williams, K. E., et al. (2006). Efficacy of varenicline, an alpha ${ }_{4}$ beta $_{2}$ nicotinic acetylcholine receptor partial agonist, Vs placebo or sustained-release bupropion for smoking cessation: a randomized controlled trial. Journal of the American Medical Association, 296, 56-63.

Klein, D. J., \& Mcintosh, S. (2003). Quitlines in North America: evidence base and applications. The American Journal of the Medical Science, 326, 201-205.

Lancaster, T., \& Stead, L. F. (2005). Self-help interventions for smoking cessation. Cochrane Database of Systematic Reviews, CD001118.

Lancaster, T., Stead, L., Silagy, C., \& Sowden, A. (2000). Effectiveness of interventions to help people stop smoking: findings from the Cochrane Library. British Medical Journal, 321, 355-358.

Lichtenstein, E., Glasgow, R. E., Lando, H. A., Ossip-Klein, D. J., \& Boles, S. M (1996). Telephone counseling for smoking cessation: rationales and metaanalytic review of evidence. Health Education Research, 11, 243-257.

Ludman, E. J., Curry, S. J., Meyer, D., \& Taplin, S. H. (1999). Implementation of outreach telephone counseling to promote mammography participation. Health Education \& Behavior, 26, 689-702.

Marques, A. C. P. R., Campana, A., Gigliotti, A. P., Lourenço, M. T. C., Ferreira M. P., \& Laranjeira, R. (2001). Consenso sobre o tratamento da dependência de nicotina. Revista Brasileira de Psiquiatria, 23, 200-214.

Marques, A. C. P. R., \& Furtado, E. F. (2004). Intervenções breves para problemas relacionados ao álcool. Revista Brasileira de Psiquiatria, 26, 28-32.

Marques, A. C. P. R., \& Ribeiro, M. (2003). Nicotina. In R. Laranjeira (Org.), Usuários de substâncias psicoativas: abordagem, diagnóstico e tratamento (pp. 49-58). São Paulo: CREMESP/AMP.

Mazoni, C. G. (2007). Aconselhamento telefônico reativo: avaliação da intervenção breve motivacional na cessação do tabagismo. Dissertação de Mestrado, Universidade Federal de Ciências da Saúde de Porto Alegre, Rio Grande do Sul.

Mazoni, C. G., Bisch, N. K., Freese, L., Ferigolo, M., \& Barros, H. M. T. (2007) Aconselhamento telefônico reativo para cessação do consumo do tabaco: relato de caso. Aletheia, 24, 137-148.

Miller, C. L., Wakefield, M., \& Roberts, L. (2003). Uptake and effectiveness of the Australian telephone quitline service in the context of a mass media campaign. Tobacco Control, 2, 53-58.

Miller, W. R. (1985). Motivation for treatment: a review with special emphasis on alcoholism. Psychological Bulletin, 98, 84-107.

Miller, W. R., \& Rollnick, S. (2001). Entrevista motivacional: preparando as pessoas para a mudança de comportamentos adictivos. Porto Alegre: Artmed.

Mitrouska, I., Bouloukaki, I., \& Siafakas, N. M. (2007). Pharmacological approaches to smoking cessation. Pulmonary Pharmacology \& Therapeutics, 20, 220-232.

Paul, C. L., Wiggers, J., Daly, J. B., Green, S., Walsh, R. A., Knight, J., et al. (2004). Direct telemarketing of smoking cessation interventions: will smokers take the call? Addiction, 99, 907-913.

Peto, R., Darby, S., Deo, H., Silcocks, P., Whitley, E., \& Doll, R. (2000). Smoking, 
smoking cessation, and lung cancer in the UK since 1950: combination of national statistics with two case-control studies. British Medical Journal, 321, 323-329

Presman, S., Carneiro, E., \& Gigliotti, A. (2005). Tratamentos não-farmacológicos para o tabagismo. Revista de Psiquiatria Clínica, 32, 267-275.

Prochasca, J. O., \& Diclemente, C. C. (1982). Transtheoretical therapy: toward a more integrative model of change. Psychotherapy: Theory, Research and Pratice, 20, 161-173.

Prochasca, J. O. \& Diclemente, C. C. (1984). Self change processes, self-efficacy and decisional balance across five stages of smoking cessation. Journal of Consulting and Clinical Psychology, 156, 131-140.

Ramelson, H. Z., Friedman, R. H., \& Ockene, J. K. (1999). An automated telephone-based smoking cessation education and counseling system. Patient Education \& Counseling, 36, 131-144.

Roche, A. M., \& Freeman, T. (2004). Brief interventions: good in theory but weak in practice. Drug and Alcohol Review, 23, 11-18.

Rollnick, S., Heather, N., \& Bell, A. (1992). Negotiating behaviour change in medical settings: the development of brief motivational interviewing. Journal of Mental Health, 1, 25-37.

Sackett, D. L., Straus, S. E., Richardson, W. S., Rosenberg, W., \& Haynes, R. B. (2003). Medicina baseada em evidências: prática e ensino. Porto Alegre: Artmed.

Scharf, D., \& Shiffman, S. (2004). Are there gender differences in smoking cessation, with and without bupropion? Pooled- and meta-analyses of clinical trials of bupropion SR. Addiction, 99, 1462-1469.

Severson, H. H., \& Harsukami, D. (1999). Smokeless tobacco cessation. Primary Care, 26, 529-551.
Shearer, J. (2007). Psychosocial approaches to psychostimulant dependence: a systematic review. Journal of Substance Abuse Treatment, 32, 41-52.

Silagy, C., Lancaster, T., Stead, L., Mant, D., \& Fowler, G. (2004). Nicotine replacement therapy for smoking cessation. Cochrane database of systematic reviews, CD000146.

Solomon, L. J., \& Flynn, B. F. (2005). Telephone support for pregnant smokers who want to stop smoking. Health promotion practice, 6, 105-108.

Stead, L. F., \& Lancaster, T. (2005). Group behaviour therapy programmes for smoking cessation. Cochrane Database System Review, CD001007.

Viegas, C. A. A., \& Reichert, J. (2004). Tratamento medicamentoso. Jornal Brasileiro de Pneumologia, 30(suplemento 2), S36-S40.

Wakefield, M., \& Borland, R. (2000). Saved by the bell: the role of telephone helpline services in the context of mass-media anti-smoking campaigns. Tobacco Control, 9, 117-119.

West, R. (2004). Assessment of dependence and motivation to stop smoking. British Medical Journal, 328, 338-339.

Willemsen, M. C., Wiebing, M., Emst, A., \& Zeeman, G. (2006). Helping smokers to decide on the use of efficacious smoking cessation methods: a randomized controlled trial of a decision aid. Addiction, 101, 1362-1363.

Woerpel, C., Wright, K. L., \& Wetter, D. W. (2006). Smoking cessation: pharmacological treatments. Behavioral Medicine, 32, 47-56.

Zhu, S. H., Anderson, C. M., Tedeschi, G. J., Rosbrook, B., Johnson, C. E., Byrd, M. et al. (2002). Evidence of real-world effectiveness of a telephone quitline for smokers. The New England Journal of Medicine, 14, 306-310.

Zhu, S. H., Tedeschi, G. J., Anderson, C. M., \& Pirce, J. P. (1996). Telephone counseling for smoking cessation: what's in a call? Journal of counseling $e$ development, 75, 93-102.

Cláudia Galvão Mazoni, mestre em Ciências Médicas pela Fundação Faculdade Federal de Ciências Médicas de Porto Alegre (FFFCMPA), é professora no curso de Psicologia da Universidade Luterana do Brasil. Endereço para correspondência: Rua Sarmento Leite, 245 - $3^{\circ}$ andar; Farmacologia/VIVAVOZ; Porto Alegre, RS; CEP: 90050-170. Tel.: (51) 3303-8764. E-mail: cmazoni@hotmail.com

Simone Fernandes, mestre em Ciências Médicas pela FFFCMPA, é supervisora do Serviço Nacional de Orientações e Informações sobre a Prevenção do Uso Indevido de Drogas (VIVAVOZ). E-mail: simone_ psicol@yahoo.com.br

Pollianna Sangalli Pierozan, mestre em Ciências Médicas pela FFFCMPA, é professora dos Cursos de Enfermagem e Agronomia do Instituto de Desenvolvimento Educacional do Alto Uruguai (Getúlio Vargas, RS). E-mail: pollipiero@gmail.com

Taís Moreira, mestre em Ciências Médicas pela FFFCMPA, é supervisora do VIVAVOZ. E-mail: tais. moreira@bol.com.br

Luana Freese, graduanda de Biomedicina, é consultora do VIVAVOZ. E-mail: lu_freese@gmail.com Maristela Ferigolo, doutora em Ciências Médicas pela Universidade Federal do Rio Grande do Sul, é coordenadora do VIVAVOZ. E-mail: mari@ufcspa.edu.br

Helena M. T. Barros, doutora em Psicofarmacologia pela Universidade Federal de São Paulo, é professora titular de Farmacologia da FFFCMPA e coordenadora do VIVAVOZ. E-mail: helenbar@ufcspa.edu.br 